\title{
A PILOT-ASSISTED EQUALISATION SCHEME FOR THE UMTS-TDD DOWNLINK WITH PARTIAL LOADING
}

\author{
Mahmoud Hadef and Stephan Weiss \\ School of Electronics \& Computer Science \\ University of Southampton, Southampton SO17 1BJ, UK \\ $\{$ m.hadef, s.weiss\}@ecs.soton.ac.uk
}

\begin{abstract}
In this paper, we address a pilot-assisted channel equalisation strategy for the partially loaded downlink time-division duplex (TDD) component of the universal mobile telecommunication system (UMTS). The adaptation is performed by minimising a hybrid cost function based on the constant modulus (CM) criterion for all active users and a mean square error (MSE) criterion for both absent users and pilot signals. Computer simulations are used to assess the performance of the equalisation strategy under various partially loaded conditions.
\end{abstract}

Keywords: UMTS TDD, constant modulus (CM), mean square error (MSE), pilots, and partial loading.

\section{INTRODUCTION}

The time division duplex (TDD) component of the universal mobile telecommunications system (UMTS) provides a high transmission rate, an efficient use of the spectrum and a flexible capacity allocation. It has previously become the basis for the third generation (3G) standard, and high likely will be selected as the main duplex mode operation for the fourth generation (4G) [1].

In the UMTS TDD mode the transmitted users are multiplexed by orthogonal codes, which provide intrinsic protection against multi-access interference (MAI). Nevertheless, transmission over a dispersive channel destroys the mutual orthogonality of these codes, and as a result, the received and code-demultiplexed user signals are subject not only to inter-symbol interference (ISI) due to channel dispersion but also to MAI caused by the loss of code orthogonality.

A popular approach to suppress MAI and ISI on a user is the minimum output power (MOE) algorithm, blindly cancelling MAI and ISI terms but passing the desired user by code-constraints [8], which is essentially Frost's linearly constrained minimum variance beamformer [14]. For the DS-CDMA downlink, the recovery of several synchronous users at the same time exploits more knowledge of the system. Non-blind multiuser schemes, such as the least mean square (LMS) algorithm, in turn are based either on the knowledge of a pilot [13, 7] or training sequences [15]. Blind schemes have been derived using a constant modulus (CM) criterion [4, 5], and the derived algorithms either neglect the dispersiveness of the channel [5] or spreading [4, 2], whereby the later additionally require mutual decorrelation of the recovered user sequences. In [6], the FIRMERCMA algorithm, a blind scheme similar to $[4,2]$ has been developed, whereby the despreading in the DS-CDMA receiver ensures the orthogonality of the recovered sequences, and a CM criterion on all users suffices. The algorithm in [6] is however only suitable for a fully loaded system, in which all possible users are active, while a hybrid CM/MSE algorithm, appropriate for partial loading scenario, has been derived in [10]. In [12], a semi-blind strategy, based on the latter algorithm [10], has been proposed for UMTS downlink system, whereby inactive users are exploited for pilots loading.

In this paper we investigate a pilot-assisted channel equalisation strategy, similar in structure to [12], for the downlink of the UMTS TDD component under various pilot loading conditions. A number of inactive users are exploited as pilots, in order to eliminate the phase ambiguity and to enhance the system performance. In Sec. 2 the definition of a signal model is given, then the hybrid CM/MSE cost function is derived in Sec. 3. Sec. 4 presents the stochastic gradient algorithm used for the semi-blind adaptation. The performance of the proposed scheme in terms of MSE and BER are presented in Sec. 5, and finally conclusions are drawn in Sec. 6.

\section{SIGNAL MODEL}

We consider the UMTS-TDD downlink model in Fig. 1 with a maximum of $N$ (assumed 16 in the following) symbolsynchronous active users, which for simplicity are assumed to have the same rate. In the case of a partially loaded system with $K \leq N$, we assume the first $K$ users with signals $u_{l}[n], l=0(1) K-1$, to be active, and the next $N_{p} \leq N-K$ to be pilots with signals $p_{l}[n], l=0(1) N_{p}-1$ while for the remaining $N-K-N_{p}$ user signals are assumed to be 


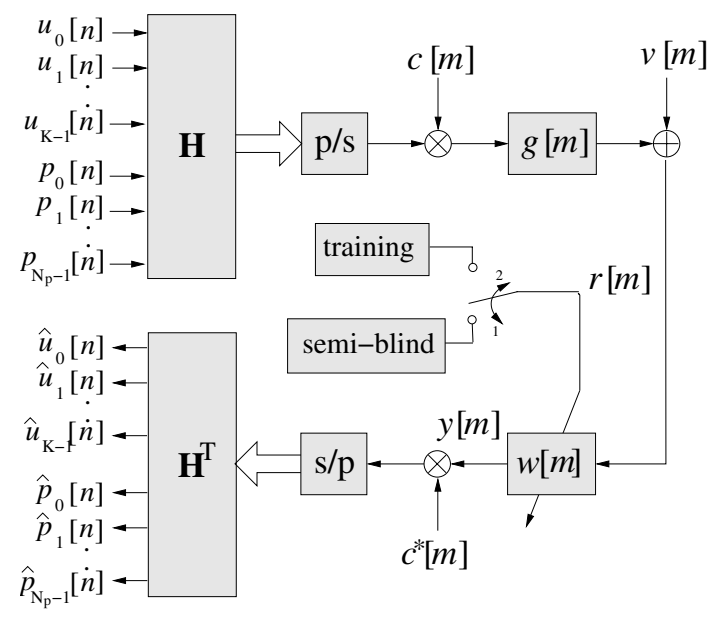

Fig. 1. Signal model.

zero. The signals $u_{l}[n]$ are code multiplexed using Walsh sequences of length $N$ extracted from a Hadamard matrix H. The resulting chip rate signal, running at $N$ times the symbol rate, is further scrambled by $c[m]$ prior to transmission over a channel with dispersive impulse response $g[m]$ and corruption by additive white Gaussian noise $v[m]$, which is assumed to be independent of the transmitted signal $s[m]$.

The dispersive channel $g[m]$ destroys the orthogonality of the Walsh codes, such that direct decoding of the received signal $r[m]$ with descrambling by $c^{*}[m]$ and code-matched filtering by $\mathbf{H}^{\mathrm{T}}$ will lead to MAI and ISI corruption of the decoded user signals $\hat{u}_{l}[n], l=0(1) N-1$. In order to reestablish orthogonality of the codes, a chip level equaliser $w[m]$ can be utilised [7]. The equalisation is performed in both midamble period and data fields. In the former by means of the training sequence at the chip rate in the MSE sense, in the latter by using a semi-blind scheme. In the following, we are concerned with the semi-blind updating of the equaliser coefficients $w[m]$.

\section{SEMI-BLIND EQUALISATION CRITERIA}

We first derive the detected user signals $\hat{u}_{l}[n]$ and the pilot signals $\hat{p}_{l}[n]$ as a function of the equaliser $w[m]$. Based on this, we state a suitable cost function based on which the equaliser can be adapted.

\subsection{Demultiplexed User and pilot Signals}

For the decoding, Walsh sequences are used as matched filters. The sequence for decoding the $l$ th user, contained in a vector $\mathbf{h}_{l}$, can be taken from an $N \times N$ Hadamard matrix,

$$
\mathbf{H}^{\mathrm{T}}=\left[\begin{array}{llll}
\mathbf{h}_{0} & \mathbf{h}_{1} & \cdots & \mathbf{h}_{N-1}
\end{array}\right]^{\mathrm{T}} .
$$

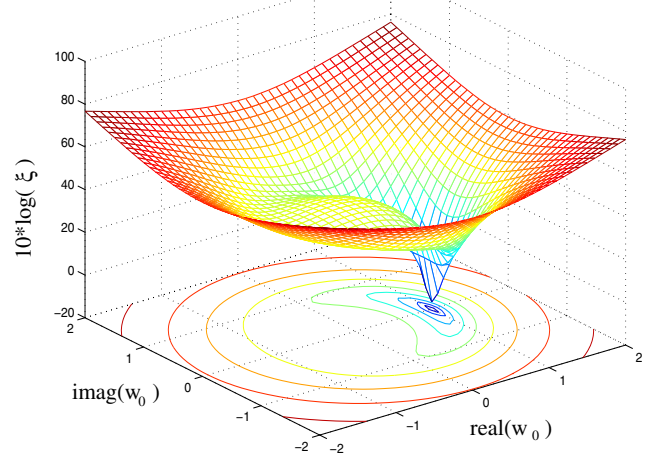

Fig. 2. Cost function $\xi$ in dependency of a single complex valued coefficient $w_{0}$, for a partially loaded system with 10 active users and 6 pilots.

The $l$ th user is thus decoded as

$$
\begin{aligned}
& \hat{u}_{l}[n]=\mathbf{h}_{l}^{\mathrm{T}} \cdot\left[\begin{array}{cc}
c^{*}[n N] & \mathbf{0} \\
c^{*}[n N-1] & \\
& \ddots \\
\mathbf{0} & c^{*}[n N-N+1]
\end{array}\right] \cdot\left[\begin{array}{c}
y[n N] \\
y[n N-1] \\
\vdots \\
y[n N-N+1]
\end{array}\right] \\
& =\tilde{\mathbf{h}}_{l}^{\mathrm{T}}[n N] \cdot\left[\begin{array}{ccc}
\mathbf{w}^{\mathrm{H}} & & \mathbf{0} \\
& \mathbf{w}^{\mathrm{H}} & \\
& & \ddots \\
& & \\
\mathbf{0} & & \mathbf{w}^{\mathrm{H}}
\end{array}\right] \cdot\left[\begin{array}{c}
r[n N] \\
r[n N-1] \\
\vdots \\
r[n N-L-N+2]
\end{array}\right]
\end{aligned}
$$

whereby the descrambling code $c^{*}[m]$ has been absorbed into a modified and now time-varying code vector $\tilde{\mathbf{h}}_{l}[n N]$, and $\mathbf{w} \in \mathbb{C}^{L}$ contains the equaliser's $L$ chip-spaced complex conjugate weights. Rearranging $\mathbf{w}$ and $\tilde{\mathbf{h}}_{l}[n N]$ yields

$$
\begin{aligned}
\hat{u}_{l}[n] & =\mathbf{w}^{\mathrm{H}} \cdot\left[\begin{array}{cc}
\tilde{\mathbf{h}}_{l}^{\mathrm{T}}[n N] & \mathbf{0} \\
\tilde{\mathbf{h}}_{l}^{\mathrm{T}}[n N] \\
& \ddots \\
\mathbf{0} & \tilde{\mathbf{h}}_{l}^{\mathrm{T}}[n N]
\end{array}\right] \cdot\left[\begin{array}{c}
r[n N] \\
r[n N-1] \\
\vdots \\
r[n N-L-N+2]
\end{array}\right] \\
& =\mathbf{w}^{\mathrm{H}} \mathbf{H}_{l}[n N] \mathbf{r}_{n N},
\end{aligned}
$$

and with similar analysis, the $l$ th pilot's demultiplexed signal can be given as

$$
\hat{p}_{l}[n]=\mathbf{w}^{\mathrm{H}} \mathbf{H}_{l}[n N] \mathbf{r}_{n N}
$$

with $\mathbf{H}_{l}[n N] \in \mathbb{C}^{L \times(N+L-1)}$ being a convolutional matrix comprising of the $l$ th either user's or pilot's modified code vector $\tilde{\mathbf{h}}^{\mathrm{T}}[n]$ and $\mathbf{r}_{n N} \in \mathbb{C}^{N+L-1}$.

\subsection{Cost Function}

Since the modulation scheme used for UMTS-TDD is mainly the quadrate phase shift keying (QPSK) (with some exceptions the 8PSK) [3], the $K$ active user signals $u_{l}[n]$ consist 
of symbols with a constant modulus $\gamma$. Therefore, by forcing all received user symbols $\hat{u}_{l}[n]$ onto the constant modulus $\gamma$ and the received pilot symbols $\hat{p}_{l}[n]$ onto the known transmitted sequences $p_{l}[n]$, a semi-blind cost function $\xi$ is proposed to adapt the equaliser weights and track any channel variations. Note that the remaining $N-K-N-p$ inactive users should be taken into consideration, otherwise the equalisation criterion are under-determined. Hence, the correct signals would not necessarily be extracted in the dispreading operation. Accordingly, the inactive users are forced to zeros in MSE sense to ensure that the overall system is fully determined.

Therefore the proposed cost function consists of three terms and is formulated as $\xi$,

$$
\begin{gathered}
\xi=\mathcal{E}\left\{\sum_{l=0}^{K-1}\left(\gamma_{l}^{2}-\left|\hat{u}_{l}[n]\right|^{2}\right)^{2}\right\}+\mathcal{E}\left\{\sum_{l=0}^{N_{p}-1}\left|p_{l}[n]-\hat{p}_{l}[n]\right|^{2}\right\}+ \\
+\mathcal{E}\left\{\sum_{l=K+N_{p}}^{N-1}\left|\hat{u}_{l}[n]\right|^{2}\right\}
\end{gathered}
$$

$\mathcal{E}\{\cdot\}$ denotes the expectation operator .The equaliser coefficients $\mathbf{w}$ can be determined such that the above cost function is minimised. However, in case where no pilot is loaded, a manifold of solutions exist for an optimum,

$$
\mathbf{w}_{\mathrm{opt}}=\arg \min _{\mathbf{w}} \xi
$$

There is no unique solution to (5), since minimising (4) is ambiguous due to an indeterminism in phase rotation.

\subsection{Phase ambiguity}

Since an ambiguity with respect to a complex rotation $e^{j \varphi}($ $\varphi \in[0 ; 2 \pi]$ ) cannot be resolved by CM criteria, this rotation invariance could be overcome by the use of the inactive codes to load pilot signals.

Example. To show how pilots overcome the phase ambiguity the following example is presented. We assume a system with $K=10$ active users and 6 pilots, over a distortion-less and delay-less channel $g=1$. Thus, as shown in Fig. 2, the cost function $\xi$ has one unique optimum solution $w_{0}=1$. Hence the phase ambiguity does not manifest itself any more.

\section{SEMI-BLIND ADAPTATION}

Simple adaption rules for the equaliser can be obtained by considering stochastic gradient descent techniques, whereby an iterative update rule is utilised for the equaliser coefficient vector $\mathbf{w}_{n}$ at time $n$,

$$
\mathbf{w}_{n+1}=\mathbf{w}_{n}-\mu \nabla \hat{\xi}
$$

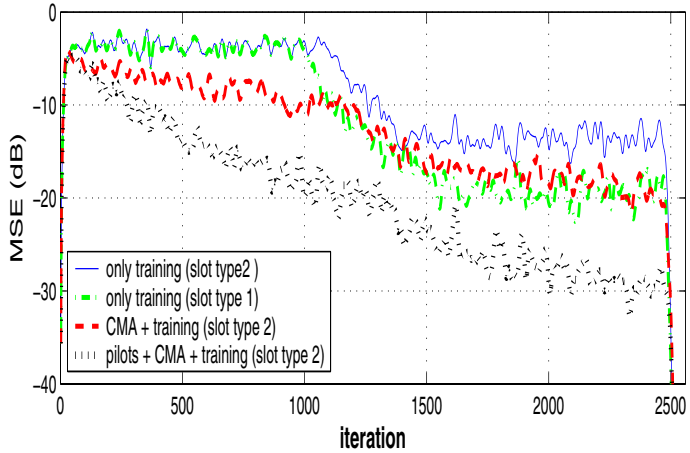

Fig. 3. MSE curves of the proposed algorithm in 10 active users UMTS-TDD system over one time slot of type 2 ( 256 chips) with and without 6 pilots and two basic chip-rate equalisation with either slot type 1(512 chips) or 2 .

where $\mu$ is the algorithm step size, and $\nabla$ the gradient operator applied to the instantaneous cost functions $\hat{\xi}$. The instantaneous cost estimates are obtained from (4) by dropping the expectation operation. The gradient term of the instantaneous cost functions can be calculated by using equations (4), (2), and (3) as follows:

$$
\begin{array}{r}
\frac{\partial \hat{\xi}}{\partial \mathbf{w}^{*}}=-2 \sum_{l=0}^{K-1}\left(\gamma^{2}-\left|\hat{u}_{l}[n]\right|^{2}\right) \mathbf{H}_{l}[n N] \mathbf{r}_{n N} \hat{u}_{l}^{*}[n]- \\
-\sum_{l=0}^{N_{p}-1} \mathbf{H}_{l}[n N] \mathbf{r}_{n N}\left(p_{l}[n]-\hat{p}_{l}[n]\right)^{*}+ \\
\sum_{l=K+N_{p}}^{N-1} \mathbf{H}_{l}[n N] \mathbf{r}_{n N} \hat{u}_{l}^{*}[n]
\end{array}
$$

The first term in the algorithm presented by (7), differs from the standard CM algorithm [9] or its extension in [4] in the inclusion of a code filtered term $\mathbf{H}_{l}[n N] \mathbf{r}_{n N}$ rather than just the equaliser input $r[n]$. A similar structure, the multiple error filtred-X LMS algorithm, has been derived in [11] for purely least mean squares based criterion.

\section{SIMULATION RESULTS}

The chosen simulation context is a downlink UMTS TDD system partially loaded by 10 active users with a spreading factor $N=16$. In the first experiment, we evaluate the convergence behaviour in terms of MSE criterion, over one time slot of either type 1 or 2 , as described [3]. The propagation environment used is the Vehicular channel A, as specified by ITU. The length of the equaliser is $L=32$, and the step size is experimentally chosen to be about an order of magnitude below the onset of divergence.

As it is shown in Fig. 3, using blind adaptation in combination with training in type 2 , the same MSE is reached 


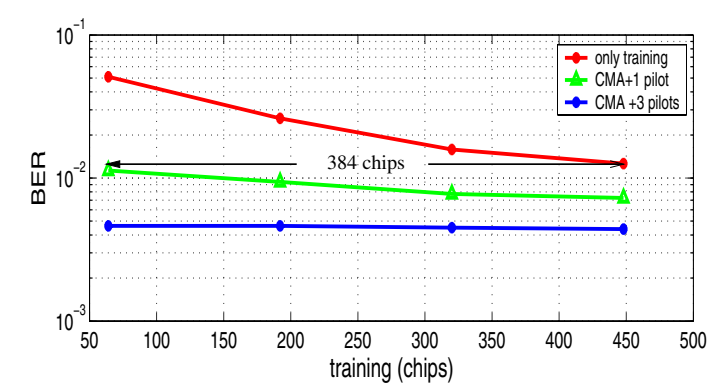

Fig. 4. Effect of pilot loading on the BER performance.

when only training in type 1 mode with longer midamble. The shortening of the midamble at no performance gain through the inclusion of the CMA is equivalent to an increase in data throughput of $13 \%$. Moreover, by loading pilots a significant MSE performance is achieved. In a second experiment, the BER is calculated for different midamble sizes, which we aim to shorten with respect to the values proposed in [3].The length of the equaliser is $L=10$, and the propagation environment used is a 3 paths quasi-time varying channel, whereby it is assumed to be invariant over each time slot. The BER is averaged over 1000 time slots, for various loading conditions.

As is depicted in Fig. 4, the performance of the pilotassisted algorithm is dramatically better than the classical training equalisation. A considerable reduction in BER and significant increase in data rate are achieved by loading pilots. For example, by loading only one pilot over a small midamble of size 64 chips, we obtain almost similar BER performance to the classical scheme where the adaptation is only performed over a seven times longer midamble, as shown in Fig. 4. Hence, 384 chips could be saved and used for data transmission. This means around $16 \%$ of data rate is gained. Moreover, by loading more pilots the BER could be reduced. For example, a reduction of at least $63 \%$ is obtained by loading three pilots for the previous short midamble, as presented in Fig. 4. However, we have noticed no further improvement in BER by adding further pilots to the scheme.

\section{CONCLUSIONS}

A pilot-assisted equalisation approach for a partially loaded UMTS-TDD downlink scenario has been presented. The algorithm presents better convergence behaviour over the basic trained equalisation even with longer training period, whereby a gain of data rate and spectrum efficiency can be achieved. It has been shown that the implementation of pilots reduce the BER and increase the data rate of the system. The proposed scheme can include pilots instead of inactive users, such the BER and data throughput are further enhanced.

\section{REFERENCES}

[1] R. Esmailzadeh, M. Nakagawa, A. Jones, “TDD-CDMA for the 4th Generation of Wireless Communication," IEEEcomms, vol. 10, Issue: 4 pp. 8-15, Aug 2003.

[2] S. Lambotharan, J. A. Chambers, and A. G. Constantinides, "Adaptive Blind Retrieval Techniques for Multi-User DSCDMA Signals," IEE Elec. Let., 35(9): 693-695, 1999.

[3] TS. 25.233, 3GPP, "Spreading and Modulation (TDD)," Third Generation Partnership Project, vol.5.3.0, April 2003.

[4] C. Papadias and A. Paulraj, "A Constant Modulus Algorithm for Multiuser Signal Separation in Presence of Delay Spread Using Antenna Arrays," IEEE SP Let., 4(6): 178-181, 1997.

[5] Y. Huang and P. M. Djurić, "Multiuser Detection of Synchronous Code-Division Multiple-Access Signals by Perfect Sampling," IEEE Trans SP, 50(7):1724-1734, 2002.

[6] S. Weiss and M. Hadef, "Blind Chip-Rate Equalisation for DS-CDMA downlink Receiver," in Asil. Conf. Sig. Sys. Comp., 2:1283 - 1287, 2003.

[7] P. Schniter and A. R. Margetts, "Adaptive Chip-Rate Equalization of Downlink Multirate Wideband CDMA," in Asilomar Conference on Signals, Systems, and Computers, Monterey, CA, November 2002, pp. 1228-1232.

[8] R. Schober, W. H. Gerstacker, and L. H. J. Lampe, "Comparison of MOE and blind LMS," IEEE Communications Letters, vol. 7, no. 5, pp. 204-206, May 2003.

[9] C. R. Johnson, P. Schniter, T. J. Endres, J. D. Behm, D. R. Brown, and R. A. Casas, "Blind Equalization Using the Constant Modulus Criterion: A Review," Proc. IEEE, 86(10): 1927-1950, 1998.

[10] M. Hadef and S. Weiss, "A Blind Synchronous Multiuser DS-CDMA Equaliser with Partial Loading ," in Int. Symp. Comms, Sig. Proc., Hammamet, Tunisia,81-84 2004.

[11] S.J. Elliott, I.M. Stothers, and P.A. Nelson, "A Multiple Error LMS Algorithm and its Application to the Active Control of Sound and Vibration," IEEE Trans. Acoustics, Speech Sig. Proc., 35(10):1423-1434, 1987.

[12] M. Hadef and S. Weiss, "A Semi-Blind Channel Equalisation Strategy for the UMTS-TDD Downlink," in 3rd workshop on the internet, telecommunication and signal processing , Adelaide, Australia, 262-267 2004.

[13] F. Petre, M. Moonen, M. Engels, B. Gyselinckx, and H.D. Man, "Pilot-Aided Adaptive CHip Rate Equalizer Receiver for Interference Suppression in DS-CDMA Forward Link," in IEEE VTC, Sept. 2000, vol. 1, pp. 303-308.

[14] O. L. Frost, III, “An Algorithm for Linearly Constrained Adaptive Array Processing," Proceedings of the IEEE, vol. 60, no. 8, pp. 926-935, August 1972.

[15] I. Stirling, D. Garcia-Alis, S. Weiss, G. Rice, and R.W. Stewart, “A Joint Adaptive MMSE Downlink Receiver," in Asilomar Conf. Sig. Sys. Comp., Nov. 2000, 2:1436-1440. 